\title{
Patient satisfaction in Dental Healthcare Centers
}

Dena A. Ali ${ }^{1}$

Correspondence: Dr. Dena A. Ali

Email: dali5@hsc.edu.kw
'Department of General Dental Practice, Faculty of Dentistry, Kuwait University, Kaifan, Kuwait

\section{ABSTRACT}

Objectives: This study aimed to (1) measure the degree of patient satisfaction among the clinical and nonclinical dental services offered at specialty dental centers and (2) investigate the factors associated with the degree of overall satisfaction. Materials and Methods: Four hundred and ninety-seven participants from five dental centers were recruited for this study. Each participant completed a self-administered questionnaire to measure patient satisfaction with clinical and nonclinical dental services. Analysis of variance, $t$-tests, a general linear model, and stepwise regression analysis was applied. Results: The respondents were generally satisfied, but internal differences were observed. The exhibited highest satisfaction with the dentists' performance, followed by the dental assistants' services, and the lowest satisfaction with the center's physical appearance and accessibility. Females, participants with less than a bachelor's degree, and younger individuals were more satisfied with the clinical and nonclinical dental services. The stepwise regression analysis revealed that the coefficient of determination $\left(R^{2}\right)$ was $40.4 \%$. The patient satisfaction with the performance of the dentists explained $42.6 \%$ of the overall satisfaction, whereas their satisfaction with the clinical setting explained $31.5 \%$ of the overall satisfaction. Conclusion: Additional improvements with regard to the accessibility and physical appearance of the dental centers are needed. In addition, interventions regarding accessibility, particularly when booking an appointment, are required.

Key words: Dental, healthcare, patient, satisfaction, Kuwait

\section{INTRODUCTION}

The exploration of customer satisfaction began in the 1970s when consumer movement was on the rise and directly related to the quality of offered services. ${ }^{[1]}$ As for the application of the consumer satisfaction in the healthcare field, in 1984, a publication measured the consumer satisfaction as a measure of the quality of life. From then, a common unanimity has developed where health services cannot be granted as high quality without the patient satisfaction measurement. Therefore, measurement of patient satisfaction is valued as a vibrant aspect of gauging the overall quality of healthcare. ${ }^{[2]}$ In the dental field, patient satisfaction can help locating the strength and weakness aspects in the dental centers and therefore, assist in elevating the quality of treatment

\begin{tabular}{|l|l|}
\hline \multicolumn{2}{|c|}{ Access this article online } \\
\hline Quick Response Code: \\
\hline
\end{tabular}

and better future planning. ${ }^{[3]}$ Patient satisfaction is a multifactorial concept that plays a major role in measuring the degree of satisfaction including the patient's educational background, lifestyle, previous medical experience, and expectations; ${ }^{[4,5]}$ therefore, the measurement of distinct magnitudes of dental health care maybe more appropriate. Consequently, in dentistry, the patient satisfaction can help to locate the strengths and weaknesses of dental services and assist in elevating the quality of treatment. ${ }^{[6]}$

This study assessed the level of patient satisfaction across 5 dental specialty centers in Kuwait. ${ }^{[7]}$ This study aimed to (1) measure the degree of patient satisfaction among the clinical dental services and

This is an open access article distributed under the terms of the Creative Commons Attribution-NonCommercial-ShareAlike 3.0 License, which allows others to remix, tweak, and build upon the work non-commercially, as long as the author is credited and the new creations are licensed under the identical terms.

For reprints contact: reprints@medknow.com

How to cite this article: Ali DA. Patient satisfaction in dental healthcare centers. Eur J Dent 2016;10:309-14.

DOI: $10.4103 / 1305-7456.184147$ 
nonclinical services offered at the dental centers and (2) investigate the factors associated with the degree of overall satisfaction.

\section{MATERIALS AND METHODS}

The sample consisted of 497 participants who were randomly selected from the 5 specialty dental centers from January to July 2015. A stratified random sampling technique was used according to the size of patients, with a 95\% confidence level $( \pm 0.05)$. Participants were equally recruited from each center, approximately 100 patients per center. Of the questionnaires distributed, 550 and 497 patients responded; the response rate was $90 \%$.

The questionnaire was designed so that patients would evaluate the dental center services. The questionnaire explored both clinical and nonclinical dental services.

The clinical dental services construct was divided into three subconstructs. (1) The dentist performance domain was highlighted by questions concerning the availability of dentists; the dentist's communication skills, demonstrated by showing an ability to listen to the patients, provide a clear explanation before treatment, and post treatment instructions. (2) The dental-assistant domain consisted of questions regarding the availability of dental assistants and their response speed to patient needs. (3) The clinical dental settings domain contained questions regarding the dentist's use of modern dental devices, the availability of required dental materials/instruments, and infection-control protocol monitoring.

The nonclinical services construct was also divided into three subconstructs. (1) Accessibility was evaluated through questions regarding accessibility to dental care, including ease of obtaining suitable appointment dates, appointment waiting time, and the waiting time before meeting the doctor on the day of the appointment. (2) The physical exterior of the reception/waiting area was assessed with two questions regarding the availability of enough seats in the waiting area and the suitability of the interior design/décor. (3) The reception staff was evaluated with questions related how well patients were welcomed at reception, the ease of obtaining suitable dates and hours for appointments through the receptionists, the speed, and accuracy of data entry.

The questionnaire also addressed patients' overall satisfaction with the treatment results, the promptness in treating patients, and the sterilization and infection-control protocols. Sociodemographic questions regarding patient gender, age, and educational level were also asked. Please refer to the questionnaire.

The questionnaire used a five-point Likert-type scale ranging from strongly satisfied (5) to strongly dissatisfied (1). The protocols outlined in the Declaration of Helsinki were followed, and the anonymity and confidentiality of the participants were assured. This questionnaire was evaluated in a pilot study of thirty participants. The questionnaire was then validated, initially by utilizing the face validity test, which confirmed that most of the items in the questionnaire have reflected the meaning of relevant concepts being tested. Further, construct validity test was used to give more insight of the actual procedures that have been tested, and it yielded 0.63 of validity coefficient, after which all necessary modifications were made. The reliability analysis of the questionnaire was performed, revealing a reliability coefficient of 0.974 .

\section{Statistical analyses}

The data analyses were conducted using SPSS (version 20; Chicago, Ill., USA). A $t$-test was used to test the differences in variables between two independent groups such as gender and education. The analysis of variance (ANOVA) was used to test the significance of the differences across more than two independent groups, as well as with regard to sequelae, as in the case of age and the dental centers. Finally, to create a model of the causal relationships among the six research variables, both a general linear model (GLM) and a stepwise regression analysis were applied. The results were considered as significant when they were associated with $P<0.05$.

\section{RESULTS}

A total of 497 questionnaires were completed. A total of 246 males and 251 females $(49.4 \% / 50.5 \%)$ participated. Approximately $62 \%$ of the respondents were between 20 and 40 years old (62\%); 18\% were younger than 20 years old, and $20 \%$ were older than 40 years old. Most of the respondents $(78.7 \%)$ had a bachelor's degree or above, whereas $21.3 \%$ had only a primary education.

The summary results indicated that all of the constructs were significant $P<0.05$ [Table 1]. Although the respondents were generally satisfied, internal 
differences were observed. Their satisfaction with the dentists' performance was highest, followed by that with the dental assistants' services. The clinical settings came third in satisfaction, followed by their overall satisfaction and their satisfaction with the reception staff. The reception area and accessibility were the least satisfactory.

Table 1 describes the associations between the patient demographic characteristics and their satisfaction with the dental services. Females were more satisfied with the clinical and nonclinical subconstructs [Table 2]. The patients with less than a bachelor's degree were more satisfied with the services [Table 3].

We also assessed the associations between age and all of the sub-constructs using an ANOVA. Patients who were 40 years old or older were less satisfied, especially with regard to the clinical settings [Table 4].

A GLM was used to identify the main effects in the satisfaction model by assessing the six created subconstructs and the sociodemographic characteristics with regard to their relationships with overall satisfaction. The dentist performance variables and the clinical settings were significantly associated with overall satisfaction. Therefore, the GLM demonstrated that the dentist performance variables and the clinical setting were the major predictors of overall satisfaction.

Additional exploration of the GLM results was performed using a stepwise regression analysis was used to provide an affirmative description of the relationship between the subconstructs. The results of the stepwise regression are shown in Table 5.

The stepwise regression analysis revealed that the coefficient of determination $\left(R^{2}\right)$ was $40.4 \%$, which refers to the amount of variation explained by the six aforementioned independent variables included in the model. This statistic also indicated that the model was adequate and provided a good fit $(P=0.000)$, and no multicollinearity problems were present given that the variance inflation factor was $<5$ for all independent variables. Patient satisfaction with dentist performance explained $42.6 \%$ of overall satisfaction, whereas that with the clinical setting explained $31.5 \%$ of overall satisfaction.

\section{DISCUSSION}

The evaluation of the quality of healthcare services has been studied as a key topic for improving healthcare

\begin{tabular}{lccccc}
\multicolumn{6}{l}{ Table 1: Summary measures } \\
\hline Construct & $\boldsymbol{n}$ & Mean & SD & Mean rank & $\boldsymbol{P}$ \\
\hline Dentist performance & 487 & 4.26 & 0.89 & 1 & $<0.001$ \\
Dental assistant & 496 & 4.15 & 0.65 & 2 & $<0.001$ \\
Clinical setting & 497 & 4.02 & 0.67 & 3 & $<0.001$ \\
Accessibility & 497 & 3.76 & 0.85 & 7 & $<0.001$ \\
Reception area & 495 & 3.76 & 0.89 & 6 & $<0.001$ \\
Reception staff & 485 & 3.81 & 0.82 & 5 & $<0.001$ \\
Overall satisfaction & 497 & 3.98 & 1.12 & 4 & $<0.001$ \\
\hline SD: Standard deviation & & & & &
\end{tabular}

\begin{tabular}{lllllc}
\multicolumn{6}{l}{ Table 2: Mean satisfaction scores by gender } \\
\hline & Gender & $\boldsymbol{n}$ & Mean & SD & $\boldsymbol{P}$ \\
\hline Dentist performance & Female & 245 & 4.35 & 0.07 & $0.002^{*}$ \\
& Male & 242 & 4.17 & 0.05 & \\
Dental assistant & Female & 248 & 4.15 & 0.04 & 0.909 \\
& Male & 248 & 4.15 & 0.03 & \\
Clinical setting & Female & 248 & 4.08 & 0.04 & $0.002^{*}$ \\
& Male & 249 & 3.95 & 0.04 & \\
Accessibility & Female & 249 & 3.85 & 0.05 & $0.002^{*}$ \\
& Male & 248 & 3.67 & 0.06 & \\
Reception area & Female & 247 & 3.84 & 0.05 & $0.005^{*}$ \\
& Male & 248 & 3.68 & 0.06 & \\
Reception staff & Female & 242 & 3.81 & 0.04 & 0.853 \\
& Male & 243 & 3.82 & 0.06 & \\
Overall satisfaction & Female & 248 & 4.08 & 0.09 & $0.004^{*}$ \\
& Male & 249 & 3.88 & 0.05 & \\
\hline
\end{tabular}

${ }^{*} P$ value is significant at $<0.05$. SD: Standard deviation

\begin{tabular}{|c|c|c|c|c|c|c|}
\hline & Education level & $n$ & Mean & SD & SE & $P$ \\
\hline \multirow[t]{2}{*}{ Dentist } & Low & 192 & 4.31 & 0.59 & 0.04 & $0.024^{x}$ \\
\hline & High & 295 & 4.22 & 1.05 & 0.06 & \\
\hline \multirow[t]{2}{*}{ Dental assistant } & Low & 198 & 4.20 & 0.71 & 0.05 & $0.00^{*}$ \\
\hline & High & 298 & 4.13 & 0.60 & 0.03 & \\
\hline \multirow[t]{2}{*}{ Clinical setting } & Low & 196 & 4.20 & 0.68 & 0.05 & 0.003 \\
\hline & High & 301 & 3.90 & 0.63 & 0.04 & \\
\hline \multirow[t]{2}{*}{ Accessibly } & Low & 197 & 3.87 & 0.88 & 0.06 & 0.042 \\
\hline & High & 300 & 3.69 & 0.82 & 0.05 & \\
\hline \multirow[t]{2}{*}{ Reception } & Low & 198 & 3.87 & 0.90 & 0.06 & 0.972 \\
\hline & High & 297 & 3.69 & 0.89 & 0.05 & \\
\hline \multirow[t]{2}{*}{ Reception staff } & Low & 191 & 3.83 & 0.76 & 0.05 & 0.465 \\
\hline & High & 294 & 3.81 & 0.85 & 0.05 & \\
\hline \multirow{2}{*}{ Overall satisfaction } & Low & 196 & 4.13 & 1.03 & 0.07 & $0.041^{*}$ \\
\hline & High & 301 & 3.89 & 1.17 & 0.06 & \\
\hline
\end{tabular}

services. Patients' opinions and views are regarded as an essential component of the quality of healthcare services. ${ }^{[8]}$

Previous studies exploring the associations between sociodemographic characteristics and satisfaction conflicted given that some studies indicated that 
Ali: Patient satisfaction in dental centers

\begin{tabular}{|c|c|c|c|c|c|c|c|}
\hline Age group & $\begin{array}{l}\text { Dentist-related } \\
\text { factors }\end{array}$ & $\begin{array}{c}\text { Dental } \\
\text { assistant }\end{array}$ & $\begin{array}{l}\text { Clinical } \\
\text { setting }\end{array}$ & Accessibility & $\begin{array}{l}\text { Reception } \\
\text { area }\end{array}$ & $\begin{array}{l}\text { Reception } \\
\text { staff }\end{array}$ & $\begin{array}{c}\text { Overall } \\
\text { satisfaction }\end{array}$ \\
\hline \multicolumn{8}{|c|}{$<20$ years old } \\
\hline Mean & 4.45 & 4.18 & 4.03 & 4.00 & 4.02 & 4.03 & 4.01 \\
\hline$n$ & 88 & 90 & 89 & 90 & 90 & 90 & 90 \\
\hline SD & 0.97 & 0.61 & 0.65 & 0.81 & 0.92 & 0.66 & 0.90 \\
\hline \multicolumn{8}{|c|}{$20-40$ years old } \\
\hline Mean & 4.26 & 4.19 & 4.04 & 3.76 & 3.81 & 4.01 & 4.00 \\
\hline$n$ & 301 & 307 & 309 & 308 & 306 & 309 & 308 \\
\hline SD & 0.92 & 0.63 & 0.67 & 0.84 & 0.88 & 0.65 & 0.87 \\
\hline \multicolumn{8}{|c|}{ >40 years old } \\
\hline Mean & 4.05 & 4.02 & 3.92 & 3.52 & 3.68 & 3.88 & 3.90 \\
\hline$n$ & 98 & 99 & 99 & 99 & 99 & 99 & 99 \\
\hline SD & 0.66 & 0.70 & 0.67 & 0.84 & 0.87 & 0.69 & 0.82 \\
\hline \multicolumn{8}{|l|}{ Total } \\
\hline Mean & 4.26 & 4.15 & 4.02 & 3.76 & 3.76 & 4.10 & 3.88 \\
\hline$n$ & 487 & 496 & 497 & 497 & 495 & 497 & 497 \\
\hline SD & 0.90 & 0.65 & 0.67 & 0.85 & 0.90 & 0.90 & 0.93 \\
\hline$P$ & 0.00 & 0.02 & 0.00 & 0.00 & 0.00 & 0.00 & 0.01 \\
\hline
\end{tabular}

\begin{tabular}{|c|c|c|c|c|}
\hline Predictor & Coefficient $(\beta)$ & $t$-ratio & $\boldsymbol{P}$ & VIF \\
\hline Constant & 0.00 & -2.02 & 0.04 & 0.00 \\
\hline Dentist performance & 0.43 & 10.52 & 0.00 & 1.27 \\
\hline Clinical setting & 0.32 & 7.77 & 0.00 & 1.27 \\
\hline
\end{tabular}

females, ${ }^{[9]}$ older patients and patients with less education ${ }^{[7]}$ were more satisfied with dental care, whereas other studies found that older and less educated patients were less satisfied than younger and highly educated patients. ${ }^{[10]}$ Subsequent studies failed to show any valid associations between patient satisfaction with dental care and either age ${ }^{[11]}$ or gender. ${ }^{[8,11]}$

The findings of this study indicate that patients older than 39 years old were less satisfied with their dental care services than younger patients. This result might be because the oral health status of younger patients is better than that of older patients, and they might require less extensive and comprehensive dental treatment and fewer follow-up visits. This effect might leave a better impression on younger patients, resulting in higher satisfaction levels. ${ }^{[12]}$ In addition, this study showed that female patients expressed higher satisfaction with dental care services than males, which corroborates another study conducted in Kuwait. ${ }^{[13]}$ Newsome and Wright suggested that this finding might be because of females' greater exposure to dental services, likely moderating their expectations. ${ }^{[14]}$

Highly educated patients were less satisfied with the dental services provided in this study. More educated patients might have more knowledge regarding the quality of dental care as well as higher expectations about dental services. ${ }^{[11]}$ However, other studies have reported different findings regarding the association between education and patient satisfaction. ${ }^{[7,14]}$

The results of this study indicated that the level of satisfaction with dental care services was considerably high for most of the tested areas, except for accessibility and the physical exterior of the reception/waiting areas. These findings corroborate an earlier study conducted in three dental centers in Kuwait. ${ }^{[13]}$

The items that scored the highest were the dentist performance and dental assistant subconstructs. This high score for the dentists might reflect the long-established continuing education program directly linked to the Ministry of Health, where attending conferences and workshops are essential to meet continuing education requirements, thereby allowing dentists to continuously update their knowledge. 


\section{CONCLUSIONS}

This study is intended to guide policymakers in the dental sector toward improving dental services. The respondents displayed high satisfaction with their dentists and the clinical setting. A need for interventions exists regarding accessibility, especially when booking an appointment and the subsequent waiting period. These interventions would promote quality, patient-focused service delivery to boost the image of the dental centers, warranting an increase in their use and helping to improve the oral health of participants, thereby promoting their well-being and quality of life.

\section{Acknowledgments}

The authors thank Professor Eino Honkala for his valuable comments and recommendations. The authors also thank the editor and the anonymous reviewers for their constructive suggestions and valuable comments.

\section{Financial support and sponsorship}

Nil.

\section{Conflicts of interest}

There are no conflicts of interest.

\section{REFERENCES}

1. Paterson J, Hoffman K. Services Marketing: Concepts, Strategies, and Cases. $4^{\text {th }}$ ed. Mason, Ohio, USA: South-Western Cengage Learning; 2011. p. 286-312.

2. NHS Management Inquiry. London: Department of Health and Social Security; 1984

3. Handelman SL, Fan-Hsu J, Proskin HM. Patient satisfaction in four types of dental practice. J Am Dent Assoc 1990;121:624-30.

4. Sitzia J, Wood N. Patient satisfaction: A review of issues and concepts. Soc Sci Med 1997;45:1829-43.

5. Carr-Hill RA. The measurement of patient satisfaction. J Public Health Med 1992;14:236-49.

6. Cohen G. Age and health status in a patient satisfaction survey. Soc Sci Med 1996;42:1085-93.

7. Ministry of Health Kuwait, Dental Administration. Annual Dental Sector Report. 31 ${ }^{\text {st }}$ ed. Kuwait: Ministry of Health; 2012. http://www. damoh.gov.kw/en/. [Last accessed on 2015 Aug 25].

8. Gürdal P, Cankaya H, Onem E, Dinçer S, Yílmaz T. Factors of patient satisfaction/dissatisfaction in a dental faculty outpatient clinic in Turkey. Community Dent Oral Epidemiol 2000;28:461-9.

9. Gopalakrishna P, Munnaleneni V. Influencing satisfaction for dental services. J Health Care Mark 1993;13:16-22.

10. Zastowny TR, Roghmann KJ, Hengst A. Satisfaction with medical care: Replications and theoretic reevaluation. Med Care 1983;21:294-322

11. Hashim R. Patient satisfaction with dental services at Ajman University, United Arab Emirates. East Mediterr Health J 2005;11:913-21.

12. Lahti S, Tuutti H, Hausen H, Käärlänen R. Patients' expectations of an ideal dentist and their views concerning the dentist they visited Do the views conform to the expectations and what determines how well they conform? Community Dent Oral Epidemiol 1996;24:240-4.

13. Al-Mudaf BA, Moussa MA, Al-Terky MA, Al-Dakhil GD, El-Farargy AE, Al-Ouzairi SS. Patient satisfaction with three dental speciality services: A centre-based study. Med Princ Pract 2003;12:39-43.

14. Reifel NM, Rana H, Marcus M. Consumer satisfaction. Adv Dent Res 1997;11:281-90 


\title{
PATIENT SATISFACTION IN DENTAL HEALTHCARE CENTERS
}

\section{Questionnaire}

\author{
Section 1: Clinical dental services
}

\begin{tabular}{|c|c|c|c|c|c|c|}
\hline Number & Degree of satisfaction about the dentists performance & & & & & \\
\hline 1 & The availability of enough dentists in the center & 5 & 4 & 3 & 2 & 1 \\
\hline 2 & The dentists' commitment to being on time and punctuality & 5 & 4 & 3 & 2 & 1 \\
\hline 3 & Ability to listen to the patients and empathize with them & 5 & 4 & 3 & 2 & 1 \\
\hline 4 & $\begin{array}{l}\text { Providing clear explanation to the patient before treatment } \\
\text { and give posttreatment instructions }\end{array}$ & 5 & 4 & 3 & 2 & 1 \\
\hline \multirow[t]{2}{*}{5} & $\begin{array}{l}\text { Dentist's clinical competence in performing the dental } \\
\text { procedure at the designated time frame }\end{array}$ & 5 & 4 & 3 & 2 & 1 \\
\hline & Degree of satisfaction regarding the dental assistants & & & & & \\
\hline 6 & The availability of enough dental assistants in the center & 5 & 4 & 3 & 2 & 1 \\
\hline 9 & The dental assistant's speed of response to the patients' needs & 5 & 4 & 3 & 2 & 1 \\
\hline 10 & The use of modern dental devices and instruments & 5 & 4 & 3 & 2 & 1 \\
\hline 11 & The availability of all the needed dental materials and instruments & 5 & 4 & 3 & 2 & 1 \\
\hline 12 & $\begin{array}{l}\text { The infection control protocol monitoring (availability of bagged sterile instruments, air/ } \\
\text { water barriers, disposable bibs, cups and suction tips, and the cleanliness of the facility) }\end{array}$ & 5 & 4 & 3 & 2 & 1 \\
\hline
\end{tabular}

Section 2: Nonclinical dental services

\begin{tabular}{|c|c|c|c|c|c|c|}
\hline Number & The sentence & $\begin{array}{l}\text { Strongly } \\
\text { satisfied }\end{array}$ & Satisfied & Neutral & Dissatisfied & $\begin{array}{l}\text { Strongly } \\
\text { dissatisfied }\end{array}$ \\
\hline & Accessibility to dental care & & & & & \\
\hline 1 & Easiness of obtaining a suiff date for appointments & 5 & 4 & 3 & 2 & 1 \\
\hline \multirow[t]{2}{*}{2} & $\begin{array}{l}\text { Waiting time before seeing the doctor } \\
\text { on the day of the appointment }\end{array}$ & 5 & 4 & 3 & 2 & 1 \\
\hline & Physical exterior of the reception/waiting area & & & & & \\
\hline 6 & Availability of enough seats in the waiting area & 5 & 4 & 3 & 2 & 1 \\
\hline \multirow[t]{2}{*}{7} & Suitability of interior design/décor of the center & 5 & 4 & 3 & 2 & 1 \\
\hline & Reception staff & & & & & \\
\hline 8 & How well you were welcomed at the reception & 5 & 4 & 3 & 2 & 1 \\
\hline 9 & $\begin{array}{l}\text { Ease of obtaining appointment } \\
\text { date through the receptionist }\end{array}$ & 5 & 4 & 3 & 2 & 1 \\
\hline 10 & Speed of response to the patient's complaints & 5 & 4 & 3 & 2 & 1 \\
\hline
\end{tabular}

Section 3: Overall satisfaction

\begin{tabular}{llllllll}
\hline \multicolumn{5}{c}{ Degree of satisfaction to the overall service } \\
$\begin{array}{l}\text { The patient's overall satisfaction } \\
\text { to the treatments results }\end{array}$ & 5 & & 3 & & 2 & 1 \\
$\begin{array}{l}\text { Getting the patient fast service } \\
\text { in case of emergencies }\end{array}$ & 5 & 4 & 3 & 4 & 2 & 1 \\
$\begin{array}{l}\text { Caring about the sterilization } \\
\text { and hygiene in the center }\end{array}$ & 5 & 4 & 3 & 4 & 2 & 1 \\
\hline
\end{tabular}

Personal data
1. Gender:
$\square$ Male
Female

2. Age: $\square<20$

$\square$ 20-40

3. Educational level: $\square$ Less than bachelor degree

Above 40

$\square$ Bachelor degree and higher 